Document downloaded from:

http://hdl.handle.net/10251/81643

This paper must be cited as:

Cantó Colomina, B.; Coll, C.; Sánchez, E. (2016). On the stability and spectral radius of a finite set of matrices. Linear and Multilinear Algebra. 64(3):353-361.

doi:10.1080/03081087.2015.1040404.

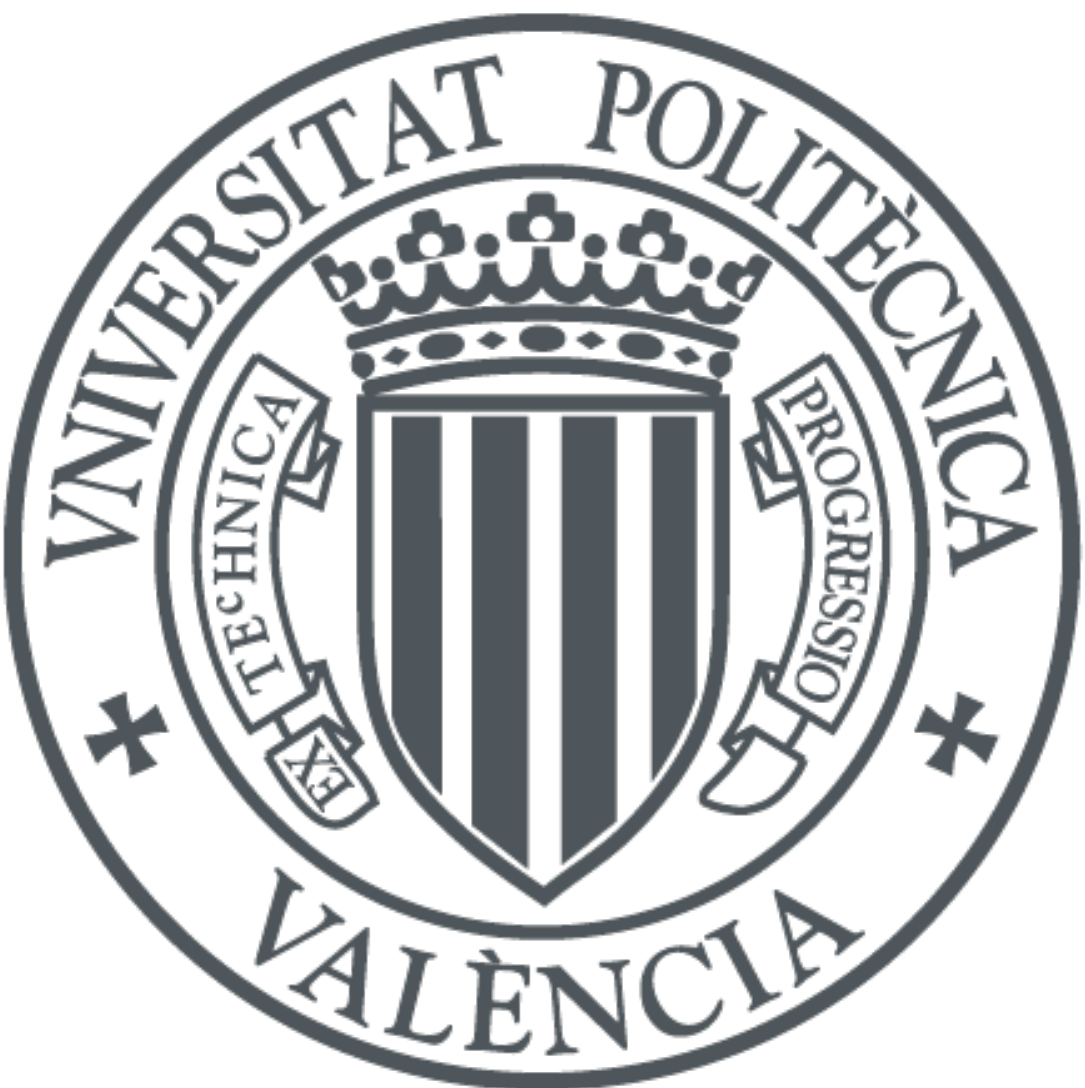

The final publication is available at

http://dx.doi.org/10.1080/03081087.2015.1040404

Copyright Taylor \& Francis

Additional Information 
To appear in Linear and Multilinear Algebra

Vol. 00, No. 00, Month 20XX, 1-9

\title{
On the stability and spectral radius of a finite set of matrices
}

\author{
B. Cantó , C. Coll and E. Sánchez \\ Instituto de Matemática Multidisciplinar, \\ Universitat Politècnica de València, \\ Camino de Vera, 14. 46022 Valencia. Spain . \\ (v3.1 released April 2014)
}

\begin{abstract}
This paper studies some problems related to the stability and the spectral radius of a finite set of matrices. A seasonal epidemic model is given to illustrate the use of the obtained results. In this example, the relationship between the obtained results and the stability of a discrete time periodic linear system is obtained.
\end{abstract}

Keywords: Nonnegative matrix, stability, spectral radius, periodic linear systems, epidemic model.

AMS Subject Classification: 15BXX; 93XX; 93A30; 93EXX

\section{Introduction}

The literature on problems related to spectral radius of nonnegative matrices is extensive. It is known, the spectral radius of matrices is a useful tool in the study of convergence of iterative methods or in the study of population dynamics, see for example [1-3]. In this paper the stability and spectral radius of a finite set of matrices is analyzed. We are interested in mathematical models exhibit seasonality, in particular, in population and epidemic models. When a population can be influenced by seasonally fluctuations it is useful to introduce periodic parameters in the design of the model and usually, the population growth is treated as a discrete event. There are several studies reported in $[4,5]$ which use periodic parameters for modelling seasonal diseases. In fact, a periodic epidemic model serves as motivation for the present study on the stability of a finite set of nonnegative matrices. As the study of the stability of matrices is related to the value of its spectral radius, we obtain some results concerning the spectral radius of special matrices obtained from the initial set of matrices.

On the other hand, the reproduction number is a threshold parameter in the study of population or epidemic models. It is defined, in the invariant case, as the spectral radius of the next generation matrix, see $[3,5,6]$. This matrix is defined as the product of a nonnegative matrix and the inverse of an M-matrix, that is, this matrix has the same structure as those used in this paper. Therefore, our results can be used to simplify numerical algorithm to calculate the estimates of the basic reproduction number and provide a method to investigate the stability of periodic epidemic models.

\footnotetext{
*Corresponding author. Email: esanchezj@mat.upv.es
} 
The paper is organized as follows. First, we give some notations, basic definitions and results. In Section 2 the characterization of the stability property of a collection of matrices is studied. For that, we obtain some conditions to ensure the stability of a collection of matrices using the spectral radius of special nonnegative matrices. In Section 3, we apply these results to epidemic models, illustrating them with some detailed examples.

We recall, see [1], that a matrix $A$ is called nonnegative if all its entries are nonnegative and it is denoted by $A \geq 0$. A square nonnegative matrix $A$ is said to be reducible if there exist a permutation matrix $P$ such that $P A P^{T}=\left(\begin{array}{cc}A_{11} & O \\ A_{21} & A_{22}\end{array}\right)$ with $A_{11}$ and $A_{22}$ square submatrices. Otherwise, $A$ is called irreducible.

On the other hand, stability of a matrix $A$ is equivalent to the condition $\rho(A)<1$ where $\rho(\cdot)$ denotes the spectral radius of a matrix, that is the maximum modulus of its eigenvalues. From the literature this property is also referred to as Schur stable matrix or convergent matrix. In [1] a characterization of this property for nonnegative matrices is given. Thus,

Lemma 1.1 A nonnegative matrix $A$ is stable if and only if $(I-A)^{-1} \geq 0$.

In general, any square matrix $A$ of the form $A=s I-B$ with $s>0$ and $B \geq 0$ is said to be an $M$-matrix if $\rho(B) \leq s$.

From Perron-Frobenius theory if $A \geq 0, \rho(A)$ is an eigenvalue of $A$ with a corresponding nonnegative Perron vector. Furthermore, if $A$ is irreducible $\rho(A)>0$ and its associated eigenvector is necessarily positive and unique up to a positive scalar factor. In [3] some results on the Perron-Frobenius theory are given. In particular, when $A+B$ is irreducible, with $\rho(A)<1$ and $B$ a nonzero nonnegative matrix, $\rho\left(B(I-A)^{-1}\right)>0$.

Before proceeding with the development of the results of the next section, let us give the following results.

Lemma 1.2 Let $M=A(I-A)^{-1}$ be with $A \geq 0$. Then $M \geq 0$ if and only if $\rho(A)<1$.

Proof. Suppose $M \geq 0$. Since $A \geq 0$ then $r=\rho(A)$ is an eigenvalue of $A$ with a nonnegative eigenvector $v$ and we have $A v=r v$ and $(I-A) v=(1-r) v$. Then, $(1-r)^{-1} \in \sigma\left((I-A)^{-1}\right)$ and $(I-A)^{-1} v=\frac{1}{1-r} v$. Hence $M v=A(I-A)^{-1} v=$ $\frac{r}{1-r} v \geq 0$ it follows that $1-\rho(A)>0$. Conversely, if $\rho(A)<1$ then $(I-A)^{-1} \geq 0$ and hence $M \geq 0$.

Lemma 1.3 Let $M=B(I-A)^{-1}$ be with $A$ and $B$ nonnegative matrices. If $A$ is an irreducible stable matrix then $\rho\left(A+\frac{B}{\rho(M)}\right)=1$.

Proof. Since $\rho(A)<1$ then $I-A$ is an $M$-matrix and $(I-A)^{-1} \geq 0$ and hence $M=B(I-A)^{-1} \geq 0$. From $A$ irreducible $r=\rho(M)>0$ is an eigenvalue of $M$ with a nonnegative eigenvector $v^{t}$. Using this vector, we have $v^{t} M=r v^{t}$ then $v^{t}(A+B / r)=v^{t}$ and hence 1 is an eigenvalue of $A+\frac{B}{r} \geq 0$. Since $A$ irreducible the matrix $A+\frac{B}{r}$ is irreducible and $\rho\left(A+\frac{B}{r}\right)=1$. 


\section{Stability of a finite collection of matrices}

Consider a set of nonzero, square nonnegative matrices $\mathcal{A}=\left\{A_{i}\right\}_{i=1}^{m}$. From now on, we denote for $i \geq 1$

$$
\Sigma_{i}(\alpha)=\sum_{j=1}^{i} \alpha_{j} A_{j}, \quad H_{i}(\alpha)=A_{i}\left(I-\Sigma_{i-1}(\alpha)\right)^{-1}, \quad M_{i}(\alpha)=A_{i}\left(I-\Sigma_{i}(\alpha)\right)^{-1}
$$

being $H_{1}(\alpha)=A_{1}$. When $\alpha=1$ these matrices will be denoted by $\Sigma_{i}, H_{i}$ and $M_{i}$, respectively. Finally, we denote $r_{i}(\alpha)=\rho\left(H_{i}(\alpha)\right)$ with $i \geq 1$ and $r_{i}=\rho\left(H_{i}\right)$.

An easy computation shows the following result.

Lemma 2.1 Consider matrices $H_{i}(\alpha)$ and $M_{i}(\alpha)$. If there exits $\left(I-H_{i}(\alpha)\right)^{-1}$ then $M_{i}(\alpha)=H_{i}(\alpha)\left(I-H_{i}(\alpha)\right)^{-1}$ and $H_{i}(\alpha)=M_{i}(\alpha)\left(I+M_{i}(\alpha)\right)^{-1}$. Furthermore, if $\rho\left(H_{i}(\alpha)\right)<1$ then $M_{i}(\alpha)=\sum_{n=1}^{\infty} H_{i}^{n}(\alpha)$.

Proof. From definition $M_{i}(\alpha)$ and $H_{i}(\alpha)$ we have

$$
\begin{aligned}
& M_{i}(\alpha)=A_{i}\left(I-\Sigma_{i}(\alpha)\right)^{-1}=A_{i}\left(I-\Sigma_{i-1}(\alpha)+A_{i}\right)^{-1}= \\
& =\left(I-\Sigma_{i-1}(\alpha)\right)^{-1}\left(I-A_{i}\left(I-\Sigma_{i-1}(\alpha)\right)^{-1}\right)^{-1}=H_{i}(\alpha)\left(I-H_{i}(\alpha)\right)^{-1} .
\end{aligned}
$$

Furthermore, if $\rho\left(H_{i}(\alpha)\right)<1$ then $\left(I-H_{i}(\alpha)\right)^{-1}=\sum_{n=0}^{\infty} H_{i}^{n}(\alpha)$ and this implies $M_{i}=\sum_{n=1}^{\infty} H_{i}^{n}(\alpha)$

The equality $H_{i}(\alpha)=M_{i}(\alpha)\left(I+M_{i}(\alpha)\right)^{-1}$ can be handled in the same way.

It is clear that we can always find a collection of nonnegative numbers $\left\{\alpha_{i}\right\}_{i=1}^{m}$ such that $\rho\left(\Sigma_{m}(\alpha)\right)<1$. The question is thus whether we can identify the conditions to ensure the stability of matrix $\Sigma_{m}$. First, let us mention a consequence of Lemma 1.2 and Lemma 2.1.

Proposition 2.2 Consider the set $\mathcal{A}$, matrices $H_{i} \geq 0$ and $M_{i}$. For each $i=$ $1, \ldots, m, \rho\left(H_{i}\right)<1$ if and only if $M_{i} \geq 0$.

Proof. For each $i=1, \ldots, m$, suppose $\rho\left(H_{i}\right)<1$. Using the relations given in Lemma 2.1 we have $M_{i}=\sum_{n=1}^{\infty} H_{i}^{n} \geq 0$. Conversely, suppose $M_{i} \geq 0$. As $H_{i} \geq 0$ using Lemma $1.2 \rho\left(H_{i}\right)<1$.

Now, we give a relation between the spectral radius of matrices $H$ and $M$.

Proposition 2.3 Consider the set $\mathcal{A}$, matrices $H_{i} \geq 0$ and $M_{i}$. For each $i=$ $1, \ldots, m$, if $\rho\left(H_{i}\right)<1$, then

$$
\rho\left(M_{i}\right)=\frac{\rho\left(H_{i}\right)}{1-\rho\left(H_{i}\right)} .
$$

Proof. For each $i=1, \ldots, m$, if $\rho\left(H_{i}\right)<1$ by Proposition 2.2 we have $M_{i} \geq 0$. 
Using the relations given in Lemma 2.1 we have

$$
\rho\left(M_{i}\right) \geq \frac{\rho\left(H_{i}\right)}{1-\rho\left(H_{i}\right)} \text { and } \rho\left(H_{i}\right) \geq \frac{\rho\left(M_{i}\right)}{1+\rho\left(M_{i}\right)} .
$$

Then $\rho\left(H_{i}\right)\left(1+\rho\left(M_{i}\right)\right) \geq \rho\left(M_{i}\right)$ and $\rho\left(H_{i}\right) \geq \rho\left(M_{i}\right)\left(1-\rho\left(H_{i}\right)\right)$. Hence $\rho\left(M_{i}\right) \leq$ $\frac{\rho\left(H_{i}\right)}{1-\rho\left(H_{i}\right)}$ and $\rho\left(M_{i}\right)=\frac{\rho\left(H_{i}\right)}{1-\rho\left(H_{i}\right)}$.

In the next result we give some conditions to obtain a lower and an upper bound for the spectral radius of matrix $\Sigma_{m}$.

Proposition 2.4 Consider the set $\mathcal{A}$, with $A_{1}$ irreducible and matrices $H_{i}$. Let $s \in\{1, \ldots, m\}$ be then the following relations hold:

(i) If $\rho\left(H_{i}\right)<1, i=1, \ldots, s$ then $\rho\left(H_{s}\right)<\rho\left(\Sigma_{s}\right)<1$.

(ii) If $\rho\left(H_{i}\right)<1, i=1, \ldots, s-1$ and $\rho\left(H_{s}\right)>1$ then $1<\rho\left(\Sigma_{s}\right)<\rho\left(H_{s}\right)$.

Proof. (i) The proof is by induction on $s$. Let $s=2$ and $A_{1}$ is an irreducible stable matrix. Construct $H_{2}=A_{2}\left(I-A_{1}\right)^{-1} \geq 0$ then $r_{2}=\rho\left(H_{2}\right)>0$. Using Lemma 1.3 $\rho\left(A_{1}+\frac{A_{2}}{r_{2}}\right)=1$ and $\rho\left(r_{2} A_{1}+A_{2}\right)=r_{2}$.

By hypothesis $r_{2}<1$, then

$$
A_{1}+A_{2} / r_{2}>\Sigma_{2}=A_{1}+A_{2}>r_{2} A_{1}+A_{2}
$$

and hence, $1>\rho\left(\Sigma_{2}\right)>r_{2}$.

Assume the above relation holds for $s-1$, we will prove it for $s$. From $r_{i}<$ $1, i=1, \ldots, s-1$ then $\rho\left(\Sigma_{s-1}\right)<1$ and as $A_{1}$ is irreducible $\Sigma_{s-1}$ is an irreducible stable matrix. Since $H_{s} \geq 0$ then $r_{s}=\rho\left(H_{s}\right)>0$. Using Lemma 1.3 we have $\rho\left(\Sigma_{s-1}+\frac{A_{s}}{r_{s}}\right)=1$ and $\rho\left(r_{s} \Sigma_{s-1}+A_{s}\right)=r_{s}$. As $r_{s}<1$,

$$
\Sigma_{s-1}+A_{s} / r_{s}>\Sigma_{s}>r_{s} \Sigma_{s-1}+A_{s}
$$

and hence $1>\rho\left(\Sigma_{s}\right)>r_{s}$.

(ii) For $i=1, \ldots, s-1, \rho\left(H_{i}\right)<1$ and suppose $r_{s}=\rho\left(H_{s}\right)>1$. Using Lemma 1.3 we have $\rho\left(\Sigma_{s-1}+\frac{A_{s}}{r_{s}}\right)=1$ and $\rho\left(r_{s} \Sigma_{s-1}+A_{s}\right)=r_{s}$. Since $r_{s}>1$,

$$
\Sigma_{s-1}+A_{s} / r_{s}<\Sigma_{s}<r_{s} \Sigma_{s-1}+A_{s}
$$

and hence, $1<\rho\left(\Sigma_{s}\right)<r_{s}$.

We will show next that the nonnegativity of matrices $\left\{M_{i}\right\}_{i=1}^{m}$ implies the stability of the matrices $\left\{H_{i}\right\}_{i=1}^{m}$ and $\left\{\Sigma_{i}\right\}_{i=1}^{m}$.

Proposition 2.5 Consider the set $\mathcal{A}$ and matrices $M_{i}$. Let $s \in\{1, \ldots, m\}$ be,

$$
\text { if } M_{i} \geq 0, i=1, \ldots, s \text { then } \rho\left(H_{i}\right)<1, i=1, \ldots, s \text {. }
$$

Proof. The proof is by induction on $s$. Let $s=1$, suppose $M_{1} \geq 0$. Since $M_{1}=$ $A_{1}\left(I-A_{1}\right)^{-1}$ and $A_{1} \geq 0$ using Lemma 1.2 we have $\rho\left(A_{1}\right)=\rho\left(H_{1}\right)<1$.

For $s=2$, suppose $M_{1} \geq 0$ and $M_{2} \geq 0$. Since $M_{2}=H_{2}\left(I-H_{2}\right)^{-1}$ with $H_{2}=A_{2}\left(I-A_{1}\right)^{-1}$ and $\rho\left(A_{1}\right)<1$, then $H_{2} \geq 0$. Using Lemma 1.2 we have $\rho\left(H_{2}\right)<1$ and hence $\left(I-H_{2}\right)^{-1} \geq 0$. 
Assume the above relation holds for $s-1$ we will prove it for $s$. For $s$, since assumption holds for $s-1$ then $\rho\left(\Sigma_{s-1}\right)<1$ and $H_{s} \geq 0$. Suppose $M_{s}=H_{s}(I-$ $\left.H_{s}\right)^{-1} \geq 0$ with $H_{s}=A_{s}\left(I-\Sigma_{s-1}\right)^{-1}$. Using Lemma 1.2 we have $\rho\left(H_{s}\right)<1$ and hence $\left(I-H_{s}\right)^{-1} \geq 0$.

Proposition 2.6 Consider the set $\mathcal{A}$ and matrices $M_{i}$. Let $s \in\{1, \ldots, m\}$ be,

$$
\text { if } M_{i} \geq 0, i=1, \ldots, s \text { then } \rho\left(\Sigma_{i}\right)<1, i=1, \ldots, s \text {. }
$$

Furthermore, $\rho\left(H_{i}\right) \leq \rho\left(\Sigma_{i}\right), i=1, \ldots, s$.

Proof. The proof is by induction on $s$. For $s=2$, suppose $M_{1} \geq 0$ and $M_{2} \geq 0$. From Proposition 2.5 we have $\rho\left(A_{1}\right)<1$ and $\rho\left(H_{2}\right)<1$ then $\left(I-A_{1}\right)^{-1} \geq 0$ and $\left(I-H_{2}\right)^{-1} \geq 0$. Consider

$$
I-\left(A_{1}+A_{2}\right)=\left(I-A_{2}\left(I-A_{1}\right)^{-1}\right)\left(I-A_{1}\right)
$$

using the definition of $M_{2}=A_{2}\left(I-A_{1}-A_{2}\right)^{-1}$, the matrix $I-\Sigma_{2}$ is invertible and its inverse is

$$
\left(I-\Sigma_{2}\right)^{-1}=\left(I-A_{1}\right)^{-1}\left(I-H_{2}\right)^{-1} \geq 0
$$

hence $\rho\left(\Sigma_{2}\right)<1$.

Assume the above relation holds for $s-1$ we will prove it for $s$. Since $\rho\left(\Sigma_{s-1}\right)<1$ then $\left(I-\Sigma_{s-1}\right)^{-1} \geq 0$. Consider

$$
I-\left(\Sigma_{s-1}+A_{s}\right)=\left(I-A_{s}\left(I-\Sigma_{s-1}\right)^{-1}\right)\left(I-\Sigma_{s-1}\right)
$$

using the definition of $M_{s}=A_{s}\left(I-\Sigma_{s-1}-A_{s}\right)^{-1}$, the matrix $I-\Sigma_{s}$ is invertible and its inverse is

$$
\left(I-\Sigma_{s}\right)^{-1}=\left(I-\Sigma_{s-1}\right)^{-1}\left(I-H_{s}\right)^{-1} \geq 0 .
$$

Hence $\rho\left(\Sigma_{s}\right)<1$.

Finally, we prove the last relation only for $s$, in the same way we can prove the other cases. If $r_{s}=\rho\left(H_{s}\right)=0$ is easily seen $\rho\left(\Sigma_{s}\right) \geq 0$. If $r_{s} \neq 0$ then $r_{s} \in \sigma\left(r_{s} \Sigma_{s-1}+A_{s}\right)$ and since $r_{s}<1$ we have $r_{s} \leq \rho\left(r_{s} \Sigma_{s-1}+A_{s}\right) \leq \rho\left(\Sigma_{s}\right)$.

Note that, the above result is not a direct consequence of Proposition 2.2 and Proposition 2.4 because it has not necessarily $A_{1}$ irreducible.

From the previous result and the nonnegativity of the matrices of the set $\mathcal{A}$ it follows that if there exits $s \in\{1, \ldots, m\}$ such that $\rho\left(H_{s}\right)>1$ then $\rho\left(\Sigma_{m}\right)>1$.

\subsection{Matrices in Frobenius normal form}

Many natural populations are composed of individuals whose birth and mortality rates differ depending on age, sex and genetics, this means that the population is distributed according to a certain structure which implies that the matrix used in the model must have that structure.

Recall that, if $A \geq 0$ is a reducible matrix it can be reduced further via permutation similarity to a block triangular form, called Frobenius normal form of $A$, where each diagonal block is square and is either irreducible or a $1 \times 1$ null 
matrix. An irreducible matrix consists of one block in the Frobenius normal form. The Frobenius normal form of a nonnegative matrix plays an important role in the graph theory and in age-structured population models and it is a useful tool to study the spectral properties of reducible matrices.

In [1] is shown as a reducible matrix can be transformed into the Frobenius normal form using similarity transformations. Thus, the spectral radius of the Frobenius normal form coincides with the spectral radius of the initial reduced matrix. It should be noted that use the Frobenius normal form to study the stability property takes advantage of the fact that the elements below the main diagonal are not of interest.

Denote by $\mathcal{M}_{l}$ the set of all lower triangular block matrices of $l \times l$ blocks where each block of the main diagonal is square of size $n_{i} \times n_{i}, i=1, \ldots, l$ and $\mathcal{M F}_{l}$ denotes the subset of all matrices of $\mathcal{M}_{l}$ in Frobenius normal form.

Consider a set of nonnegative matrices $\mathcal{A F}=\left\{A_{i} \in \mathcal{M F}_{l}\right\}_{i=1}^{m}$, where $\operatorname{diag}\left(A_{i}\right)=$ $\left(A_{1}^{(i)}, \cdots, A_{l}^{(i)}\right)$ with $A_{j}^{(i)}$ irreducible, $j=1, \ldots, l$ and $i=1, \ldots, m$.

Note that, from the blocks along the diagonal of the Frobenius normal form, for each $i=1, \ldots, m$, the matrix $\Sigma_{i} \in \mathcal{M F}_{l}$. The matrices $H_{i}$ and $M_{i}$ are in the set $\mathcal{M}_{l}$ with $\operatorname{diag}\left(H_{i}\right)=\left(H_{1}^{(i)}, \cdots, H_{l}^{(i)}\right)$ and $\operatorname{diag}\left(M_{i}\right)=\left(M_{1}^{(i)}, \cdots, M_{l}^{(i)}\right)$, respectively.

In the next result we obtain a convergence criteria for the corresponding matrices $\Sigma_{s}, s=1, \ldots, m$.

Proposition 2.7 Consider the set $\mathcal{A} F$ and its $H_{i}$ matrices. Let $s \in\{1, \ldots, m\}$ be, then the following relations hold:

(i) $\rho\left(H_{j}^{(i)}\right)<1, i=1, \ldots, s$ and $j=1, \ldots, l$ then $\rho\left(H_{s}\right) \leq \rho\left(\Sigma_{s}\right)<1$.

(ii) $\rho\left(H_{j}^{(i)}\right)<1, i=1, \ldots, s-1, j=1, \ldots, l$ and $\rho\left(H_{j}^{(s)}\right)>1 j=1, \ldots, l$ then $\rho\left(H_{s}\right) \geq \rho\left(\Sigma_{s}\right) \geq 1$.

Proof. (i) The proof is by induction on $s$ as in Proposition 2.4. Assume the assertion holds for $s-1$ we will prove it for $s$.

Since the assertion holds for $s-1$ then $\rho\left(H_{s-1}\right)<\rho\left(\Sigma_{s-1}\right)<1$ and by construction $H_{s} \in \mathcal{M}_{l}$ with $\rho\left(H_{s}\right)=\max _{j=1, \ldots, l}\left(r_{j}^{s}\right)$ being $r_{j}^{s}=\rho\left(H_{j}^{(s)}\right)$. Using the properties of the matrices involved in definition of $H_{j}^{(s)}=A_{j}^{(s)}\left(I-\sum_{j}^{(s-1)}\right)^{-1}$ we have $r_{j}^{s}>0$ for $j=1, \ldots, l$.

Without loss of generality we can assume $\rho\left(H_{s}\right)=r_{1}^{s}$ and it is clear that $r_{j}^{s} \leq r_{1}^{s}$ for $j=1, \ldots, l$. Using Lemma $1.3 \rho\left(\Sigma_{j}^{(s-1)}+\frac{A_{j}^{(s)}}{r_{j}^{s}}\right)=1$ and $\rho\left(r_{j}^{s} \Sigma_{j}^{(s-1)}+A_{j}^{(s)}\right)=r_{j}^{s}$. From $r_{j}^{s} \leq r_{1}^{s}$ for $j=1, \ldots, l$ we have

$$
\rho\left(\Sigma_{j}^{(s-1)}+\frac{A_{j}^{(s)}}{r_{1}^{s}}\right) \leq \rho\left(\Sigma_{j}^{(s-1)}+\frac{A_{j}^{(s)}}{r_{j}^{s}}\right)=1 .
$$

From above relation and since $\rho\left(\Sigma_{1}^{(s-1)}+\frac{A_{1}^{(s)}}{r_{1}^{s}}\right)=1$ we have

$$
\rho\left(\Sigma_{s-1}+\frac{A_{s}}{r_{1}^{s}}\right)=\max _{j=1, \ldots, l} \rho\left(\Sigma_{j}^{(s-1)}+\frac{A_{j}^{(s)}}{r_{1}^{s}}\right)=1 .
$$

Since $r_{1}^{s}<1, \rho\left(\Sigma_{s-1}+\frac{A_{s}}{r_{1}^{s}}\right) \geq \rho\left(\Sigma_{s}\right) \geq \rho\left(r_{1}^{s} \Sigma_{s-1}+A_{s}\right)$. And hence, $1 \geq \rho\left(\Sigma_{s}\right) \geq r_{s}$, since $\rho\left(r_{1}^{s} \Sigma_{s-1}+A_{s}\right) \geq \rho\left(r_{1}^{s} \Sigma_{1}^{(s-1)}+A_{1}^{(s)}\right)=r_{1}^{s}$. 
Suppose $\rho\left(\Sigma_{s}\right)=1$ without loss of generality we can assume $\rho\left(\Sigma_{1}^{(s)}\right)=1$. Since the assertion holds for $s-1$ then $\rho\left(H_{s-1}\right) \leq \rho\left(\Sigma_{s-1}\right)<1$, in particular $\rho\left(\Sigma_{1}^{(s-1)}\right)<1$ and $\Sigma_{1}^{(s-1)} \geq 0$ and $\Sigma_{1}^{(s)} \geq 0$ are irreducible. Since $\rho\left(\Sigma_{1}^{(s)}\right)=1$ using Proposition 2.4 we have $\rho\left(H_{1}^{(s)}\right)=1$. This contradicts our assumption. Then $\rho\left(\Sigma_{s}\right)<1$.

(ii) Denoting by $r_{j_{0}}^{s}=\min _{j=1, \ldots, l}\left\{r_{j}^{s}\right\}$ we have

$$
1=\rho\left(\Sigma_{j}^{(s-1)}+\frac{A_{j}^{(s)}}{r_{j}^{s}}\right) \leq \rho\left(\Sigma_{j}^{(s-1)}+\frac{A_{j}^{(s)}}{r_{j_{0}}^{s}}\right) \leq \rho\left(\Sigma_{s-1}+\frac{A_{s}}{r_{j_{0}}^{s}}\right) .
$$

Moreover $\rho\left(r_{j_{0}}^{s} \Sigma_{j}^{(s-1)}+A_{j}^{(s)}\right) \leq r_{j}^{s}$ for all $j=1, \ldots, l$. Thus,

$$
\rho\left(r_{j_{0}}^{s} \Sigma_{s-1}+A_{s}\right) \leq \max _{j=1, \ldots, l} r_{j}^{s}
$$

Then $1 \leq \rho\left(\Sigma_{s}\right) \leq \rho\left(H_{s}\right)$.

Note that if in the above proposition the condition $\rho\left(H_{j}^{(s)}\right)>1 j=1, \ldots, l$ is changed by the weaker condition $\rho\left(H_{s}\right)>1$, then we can only assert that $\rho\left(\Sigma_{s}\right) \geq 1$. This is straightforward assuming without loss of generality that $\rho\left(H_{s}\right)=r_{1}^{s}>1$. Hence, $r_{j}^{s} \leq r_{1}^{s}, j=1, \ldots, l$, and applying the same method as in the above proposition we have

$$
\rho\left(\Sigma_{s-1}+\frac{A_{s}}{r_{1}^{s}}\right)=\max _{j=1, \ldots, l}\left\{\rho\left(\Sigma_{j}^{(s-1)}+\frac{A_{j}^{(s)}}{r_{1}^{s}}\right)\right\}=1 .
$$

And, as in this case $r_{1}^{s}>1$, then $\rho\left(\Sigma_{s}\right) \geq \rho\left(\Sigma_{s-1}+\frac{A_{s}}{r_{1}^{s}}\right)=1$.

It is important to focus our attention on the difference between the obtained results considering the reducible or irreducible case. In the reducible case, some inequalities of the Proposition 2.6 are not strict. To illustrate this fact it is sufficient consider the following example. Consider the reducible matrices

$$
A_{1}=\left(\begin{array}{ccc}
0.33 & 0 & 0 \\
0.11 & 0.11 & 0 \\
0 & 0 & 0
\end{array}\right) \quad A_{2}=\left(\begin{array}{ccc}
0 & 0 & 0 \\
0.11 & 0.16 & 0 \\
0 & 0 & 0.33
\end{array}\right)
$$

and we check $\rho\left(H_{2}\right)=\rho\left(A_{2}\left(I-A_{1}\right)^{-1}\right)=0.33=\rho\left(\Sigma_{2}\right)<1$.

Obviously, some results developed in this section related to the stability of matrices $\Sigma_{s}, s=1, \ldots, m$ have computational advantage over the more traditional techniques. In particular, to check that a matrix is not negative is less computationally expensive to compute the spectral radius of a matrix.

\section{Application to a seasonal epidemic model}

We can find an important application of this study in the epidemic model approach. For example, we consider an SIR model, [7, 8], with three states: Susceptible individuals $S$ (number of individuals not yet infected or susceptible to the disease), Infected individuals $I$ (number of individuals who have infected with the disease) and Removed individuals $R$, (those individuals who have been infected and then 
removed from the disease). Births $\beta$ are added to $S$ state and let $p, q$ and $r$ be the survival rate of the $S, I$ and $R$ states, respectively. Let $x=(S, I, R)$ be the state vector.

From now on, we denote the periodic SIR model as $x(k+1)=E(k) x(k)$. We focus on an SIR epidemic model with periodic coefficients, which is thought to be realistic for the seasonal fluctuations in disease prevalence. In this model we have considered seasonality in the rate of susceptible individuals becoming infectious individual.

On the other hand, the basic reproduction number is used by some mathematical modelling in studying the spread of infection agent. Thus we aim to find a small set of model components that determine the dynamics of infectious contacts made by an infective in a wholly susceptible population. It is clear, that we must use the following system $I(k+1)=(T+F(k)) I(k)$. We consider an example where the influence of the diseases differs according to the case of juvenile individuals or adults individuals. Then, the infected population is divided into two classes: $I(k)=\left(I_{j}(k) I_{a}(k)\right)^{T}$, where juvenile and adult individuals will be denoted by the subscript " $\mathrm{j}$ " and "a" respectively. In this case, the periodic system is given by $I(k+1)=(T+F(k)) I(k)$, with $F(k+N)=F(k)$ with $N=2$ and

$$
T=\left(\begin{array}{cc}
q_{j}\left(1-\gamma_{j}-s\right) & 0 \\
q_{j} s & q_{a}\left(1-\gamma_{a}\right)
\end{array}\right) \quad \text { and } \quad F(k)=\left(\begin{array}{cc}
h_{j}(k) & 0 \\
0 & h_{a}(k)
\end{array}\right)
$$

being $h(k), \gamma$ and $s$ the rate of susceptible individuals becoming infectious individual, the rate of infectious individual becoming removed individual and the rate of infected juvenile individuals becoming infected adult individuals, respectively.

To know if the periodic system $I(k+1)=(T+F(k)) I(k)$ is or not stable it is sufficient to study the $\rho\left((T+F(1))(T+F(0))\right.$. That is, if $\rho\left(T^{2}+F(1) T+\right.$ $T F(0)+F(1) F(0))<1$. It is clear that we can use the propositions given in Section 2 to study the stability property since the collection of matrices of this case $\mathcal{A} F=\left\{T^{2}, F(1) T, T F(0) F(1) F(0)\right\} \in \mathcal{M F}_{2}$. In particular we have

$$
\begin{aligned}
& M_{1}=T^{2}\left(I-T^{2}\right)^{-1} \\
& M_{2}=F(1) T\left(I-T^{2}-F(1) T\right)^{-1} \\
& M_{3}=T F(0)\left(I-T^{2}-F(1) T-T F(0)\right)^{-1} \\
& M_{4}=F(1) F(0)\left(I-T^{2}-F(1) T-T F(0)-F(1) F(0)\right)^{-1} .
\end{aligned}
$$

For example, tacking the following dates $\left(q_{j}, q_{a}, \gamma_{j}, \gamma_{a}, s\right)=(0.9,0.7,0.1,0.5,0.1)$ and $\left(h_{j}(0), h_{j}(1), h_{a}(0), h_{a}(1)\right)=(0.4,0.1,0.3,0.08)$, it is easy to check $M_{i} \geq 0$, $i=1, \ldots, 4$ then we can assure that the periodic system is stable, $\rho((T+F(1))(T+$ $F(0))<1$.

In discrete population is used the net reproductive rate or basic reproductive number, see $[3,5,6]$, to analyze the asymptotic dynamics of the linear system. The basic reproduction number, denoted by $\mathcal{R}_{0}$ quantifies the expected number of offspring per individual over the course of its lifetime. In the periodic case this parameter is defined as $\mathcal{R}_{0}=\mathcal{R}_{0}^{e}=\rho\left(F_{e}\left(I-T_{e}\right)^{-1}\right)$, see $[4,9]$, where $F_{e}$ and $T_{e}$ are weakly cyclic matrices of index $N$ from $F(\cdot)$ and $T$, and represent the infection and the transition matrices, respectively.

From the fundamental idea underlying in the concept of basic reproduction number, we define a new measure, which helps us in the study of a threshold property associated to the stability of the system. It is easy to check that, $\left\{H(s)=F(s+1) T\left(I-T^{2}\right)^{-1}, s=0,1\right\}$ shows the distribution of all offspring accumulated during the lifespan of the population from the initial states, 
$\{I(s), s=0,1\}$. This fact motivates us to define the reduced reproduction number $\overline{\mathcal{R}}_{0}=\max \left\{\mathcal{R}_{0}(s)=\rho(H(s)), s=0,1\right\}$ as an approximation to the basic reproduction number. Note that, matrices $\{H(s), s=0,1\}$ have the same structure as the discussed in the previous section and we can use propositions of Section 2 to study this parameter.

In particular, if $\rho(H(0))>1$ then $\rho\left(T^{2}+F(1) T+T F(0)+F(1) F(0)\right)>1$ and this implies $\mathcal{R}_{0}>1$ and the epidemic starts, i.e., there is a take off of the epidemic that eventually settles down to an endemic equilibrium. To prove this requires only that $M_{2}=F(1) T\left(I-T^{2}-F(1) T\right)^{-1}$ has at least one negative entry.

In our example, tacking $\left(q_{j}, q_{a}, \gamma_{j}, \gamma_{a}, s\right)=(0.8,0.9,0.05,0.5,0.1)$ and $\left(h_{j}(0), h_{j}(1), h_{a}(0), h_{a}(1)\right)=(0.6,0.9,0.3,0.7)$ it is easy to check $M_{2}$ has negative entries

$$
M_{2}=\left(\begin{array}{lr}
-8.22 & 0 \\
-2.03 & 0.65
\end{array}\right), \rho\left(H_{1}\right)=\rho\left(T^{2}\right)=0.46<1, \rho\left(H_{2}\right)=\rho(H(0))=1.13>1 .
$$

Moreover, according with the results of Section 2,

$$
1<\rho\left(T^{2}+F(1) T\right)=1.07<\rho\left(H_{2}\right)=1.13 .
$$

Hence, $\rho((T+F(1))(T+F(0))>1$ and the epidemic starts. Clearly, this parameter has advantages over the parameter $\mathcal{R}_{0}$ when we have to perform an algorithm, since it is easier to check that a matrix is not negative than to obtain the spectral radius of the matrix $F_{e}\left(I-T_{e}\right)^{-1}$.

\section{References}

[1] Berman A. and Plemons R.J. Nonnegative Matrices in Mathematical Science. Philadelphia: SIAM; 1994.

[2] Johnson C.R. Bru R. The spectral radius of a product of nonnegative matrices. Linear Algebra Appl. 1990; 141: 227-240.

[3] Li C.K. Schneider H. Applications of Perron-Frobenius theory to population dynamics. J. Math. Biol. 2002; 44: 450-462.

[4] Bacaër N. Periodic matrix population models: growth rate, basic reproduction number; and entropy. Bulletin Math. Biol. 2009; 71: 1781-1792.

[5] Cushing J.M. Yicang Z. The net reproductive value and stability in matrix population models. Nat.Resour. Model. 1994; 8: 297-333.

[6] Cushing J.M. Ackleh A.S. A net reproductive number for periodic matrix models. J. Biol. Dyn. 2012; 6(2): 166-188.

[7] Allen L. Van Den Driessche P. The basic reproduction number in some discrete-time epidemic models. J. Difference Equ. Appl. 2008; 1-19.

[8] Cao H. Zhou Y. The basic reproduction number of discrete SIR and SEIS model with periodic parameters. DCDS-B 2013; 18 (1): 37-56.

[9] Bacaër N. Gomes M.G. On the final size of epidemics with seasonality. Bulletin Math. Biol. 2009; 71: 1954-1966. 